\title{
Completeness of multiseparable superintegrability on the complex 2-sphere
}

\author{
E. G. Kalnins \\ Department of Mathematics and Statistics, \\ University of Waikato, \\ Hamilton, New Zealand. \\ W. Miller, Jr. \\ School of Mathematics, University of Minnesota, \\ Minneapolis, Minnesota, 55455, U.S.A. \\ and G. S. Pogosyan* \\ Laboratory of Theoretical Physics, \\ Joint Institute for Nuclear Research, \\ Dubna, Moscow Region 141980, Russia
}

February 17, 2000

\begin{abstract}
The possibility that Schrödinger's equation with a given potential can separate in more than one coordinate system is intimately connected with the notion of superintegrability. Here we demonstrate how to establish a complete list of such potentials on the complex 2-sphere, using essentially algebraic means. Our approach is to classify all nondegenerate potentials that admit a pair of second order constants of the motion. Here "nondegenerate means that the potentials depend on four independent parameters. The method of proof generalizes to other spaces and dimensions. We show for the 2-sphere

${ }^{*}$ Work supported in part by the Russian Foundation for Basic Research under grant 98-01-00330
\end{abstract}


that all these superintegrable systems correspond to quadratic algebras, and we work out the detailed structure relations and their quantum analogs.

\section{Introduction}

It has long been known that Schrödinger's equation with certain special potentials can admit (multiplicative) separation of variables in more than one coordinate system. This is intimately related to the notion of superintegrability, $[1,2]$. This subject has been studied by a number of authors, based on the use of the corresponding differential equations that that are implied by the requirement of simultaneous separability, $[3,4,5,6,7,8,9,10,11]$. Specifically, superintegrability means that for a Schrödinger equation in dimension $N$ there exist $2 N-1$ functionally independent quantum mechanical observables (i.e., self-adjoint operators that commute with the Hamiltonian). There is an analogous concept of superintegrability for classical mechanical systems that relates to the corresponding additive separation of variables of the Hamilton-Jacobi equation. Also, if we do have simultaneous separability then the resulting constants of the motion close quadratically under repeated application of the Poisson bracket, $[12,13]$. We also know that for spaces of constant curvature separable coordinate systems of the free motion are described by quadratic elements of the corresponding first order symmetries, [14]. Although concrete examples of superintegrable systems on constant curvature spaces are easily at hand, a complete classification of all such systems has presented major difficulties. How can one be sure that all systems for free motion have been found? Once these are determined, how can one be sure that the most general additive potential term has been calculated?

In [15] we have solved the classification problem for all systems on two dimensional complex Euclidean space. Here we solve the problem for the more difficult case of the two dimensional complex sphere, including real spheres and hyperboloids as special cases. Our method is to classify all nondegenerate potentials that admit a pair of second order constants of the motion. Here "nondegenerate" means that the potentials depend on four indepen-

dent parameters. The requirement that a potential admit two constants of the motion leads to two second order partial differential equations obeyed by the potential, and the integrability conditions for these two simultaneous 
equations permit us to classify all possibilities. (We believe that this paper contains the first complete list of the possibilities, as well as a completeness proof. This is not a simple problem. For example reference [16] omits several of our cases.) The classification is greatly simplified by the equivalence of two potentials that are related by an action of the motion group $S O(3, C)$. We can prove that each nondegenerate potential is associated with a pair of constants of the motion in the classical case, and a pair of symmetry operators in the quantum case, that generate a quadratic algebra. Furthermore, we verify that there is a one-to-one correspondence between superintegrable systems and free-field symmetry operators that generate quadratic algebras. Finally, we demonstrate explicitly that superintegrability implies multiseparability, i.e., separability in more than one coordinate system.

\section{Superintegrability on the complex sphere}

We follow the approach of [15] and start by computing the possible secondorder constants of the motion for a Hamilton-Jacobi equation, with potential, on the complex sphere. (Inevitably, the only potentials that are candidates for superintegrability are those which are separable in more than one coordinate system on the two dimensional complex sphere $S_{2 C}$.) Here one considers the generators of the corresponding complex rotation group

$$
J_{1}=y p_{z}-z p_{y}, \quad J_{2}=z p_{x}-x p_{z}, \quad J_{3}=x p_{y}-y p_{x} .
$$

The Hamilton-Jacobi equation is

$$
H^{\prime}=J_{1}^{2}+J_{2}^{2}+J_{3}^{2}+V^{\prime}(x, y, z)=E^{\prime},
$$

where $x^{2}+y^{2}+z^{2}=1$. To sart with, it is convenient to make use of the natural embedding of the complex sphere in complex Euclidean 3-space. Consider the Hamiltonian

$$
H=p_{x}^{2}+p_{y}^{2}+p_{z}^{2}+V(x, y, z)
$$

in $E_{3 C}$, where $V=r^{-2} V^{\prime}$ with $r^{2}=x^{2}+y^{2}+z^{2}$. Then

$$
H=r^{-2}\left(x p_{x}+y p_{y}+z p_{z}\right)^{2}+r^{-2} H^{\prime},
$$

and we can identify the constants of the motion $L$ for $H^{\prime}$ with constants of the motion for $H$ such that

$$
\left\{L, x p_{x}+y p_{y}+z p_{z}\right\}=\left\{L, r^{2}\right\}=0 .
$$


Here of course

$$
\{f, g\}=\sum_{j=1}^{3}\left(\frac{\partial f}{\partial p_{j}} \frac{\partial g}{\partial x_{j}}-\frac{\partial f}{\partial x_{j}} \frac{\partial g}{\partial p_{j}}\right), \quad\left(x_{1}, x_{2}, x_{3}\right)=(x, y, z) .
$$

We now determine the conditions that the function

$$
L=\sum_{j, k=1}^{3} a^{j k}(x, y) p_{j} p_{k}+W(x, y, z)=\ell+W, \quad a^{j k}=a^{k j},
$$

must satisfy to be a constant of the motion for $H^{\prime}$. The conditions (4) imply

$$
\sum_{j} x_{j} \partial_{x_{j}} W=0
$$

Furthermore, these conditions and the requirement $\{H, L\}=0$ imply that the quadratic terms in $L$ are expressible as a second order polynomial in the $J_{k}$,

$$
\ell=A J_{3}^{2}+B J_{1}^{2}+C J_{2}^{2}+D J_{3} J_{1}+E J_{3} J_{2}+F J_{1} J_{2}
$$

and that

$$
\partial_{x_{j}} W=\sum_{k=1}^{3} a^{j k} \partial_{x_{k}} V
$$

The integrability conditions

$$
\partial_{x_{k}}\left(\partial_{x_{j}} W\right)=\partial_{x_{j}}\left(\partial_{x_{k}} W\right), \quad k \neq j
$$

are

$$
\begin{aligned}
\left(-2 A x y+D y z+E x z-F z^{2}\right)\left(V_{y y}-V_{x x}\right) & +\left(-2 C x z-D y^{2}+E y x+F y z\right) V_{y z} \\
+2\left(A\left[y^{2}-x^{2}\right]+[C-B] z^{2}+D x z-E y z\right) V_{x y} & -\left(-2 B y z+D x y-E x^{2}+F z x\right) V_{x z} \\
=(-6 A y+3 E z) V_{x}+(6 A x-3 D z) V_{y} & +(3 D y-3 E x) V_{z}, \\
\left(-2 C x z-D y^{2}+E y x+F y z\right)\left(V_{z z}-V_{x x}\right) & +\left(-2 A x y+D y z+E x z-F z^{2}\right) V_{y z} \\
+2\left(C\left[z^{2}-x^{2}\right]+[A-B] y^{2}-E y z+F x y\right) V_{z x} & -\left(-2 B y z+D x y-E x^{2}+F z x\right) V_{y x} \\
=(-6 C z+3 E y) V_{x}+(-3 E x+3 F z) V_{y} & +(6 C x-3 F y) V_{z}, \\
\left(-2 B y z+D x y-E x^{2}+F z x\right)\left(V_{z z}-V_{y y}\right) & +\left(-2 A x y+D y z+E x z-F z^{2}\right) V_{x z} \\
+2\left([A-C] x^{2}+B\left[z^{2}-y^{2}\right]-D x z+F x y\right) V_{z y} & -\left(-2 C x z-D y^{2}+E y x+F y z\right) V_{x y} \\
=(-3 D y+3 F z) V_{x}+(-6 B z+3 D x) V_{y} & +(6 B y-3 F x) V_{z},
\end{aligned}
$$


The homogeneity requirement on the embedded potential can be expressed as

$$
x V_{x}+y V_{y}+z V_{z}=-2 V
$$

and this leads to the additional second order conditions

$$
\begin{aligned}
& x V_{x x}+y V_{x y}+z V_{x z}=-3 V_{x} \\
& x V_{x y}+y V_{y y}+z V_{y z}=-3 V_{y} \\
& x V_{x z}+y V_{z y}+z V_{z z}=-3 V_{z} .
\end{aligned}
$$

Note that here the "trival" solution for all of these equations is $V=c / r^{2}$.

One way to attack the problem of finding all superintegrable potentials on the sphere is to classify all potentials $V$ that admit two functionally independent constants of the motion and is "nondegenerate" in the sense that it depends on four arbitray constants, one of which can be considered to be the trivial constant $c$. The potential must satisfy 2 sets of equations of the form (10) and the conditions (11). These 9 equations, not all independent, enable us to solve for the second derivatives $V_{x x}, V_{y y}, V_{z z}, V_{x z}, V_{y z}$ as linear combinations of the derivatives $V_{x y}, V_{x}, V_{y}, V_{z}$. Then all higher derivatives can be expressed in term of those 4 , and integrability conditions obeyed by the higher derivatives imply linear relations between the 4 derivatives. Nondegeneracy of the potential means that at a nonsingular point $\left(x_{0}, y_{0}, z_{0}\right)$ on the sphere we can prescribe the values of $V_{x y}, V_{x}, V_{y}, V_{z}$ arbitrarily. Thus the coefficients of all linear relations between these derivatives must vanish identically for nondegenerate potentials. Similarly, the linear relations between the terms in $V_{x x}, V_{y y}, V_{z z}, V_{x z}, V_{y z}$ on the left-hand sides of the original 9 equations imply the same linear relations between $V_{x y}, V_{x}, V_{y}, V_{z}$, and these must also vanish identically. This approach to superintegrability on the sphere will prove useful in a forthcoming paper, where we study superintegrability in 3-space. However, we will adopt a simpler method for the remainder of this paper.

A second way to carry out the analysis (and the one that we shall follow) is directly in terms of coordinates $x, y, z$ on the 2-sphere where $x^{2}+y^{2}+z^{2}=1$. We shall take $x, y$ as independent variables and set $z= \pm \sqrt{1-x^{2}-y^{2}}$ with the sign depending on wether we are on the upper or lower hemisphere of $S_{2 C}$. In some formulae we will adopt the convention $(x, y, z)=\left(y_{1}, y_{2}, y_{3}\right)$. In these coordinates the generators of the complex rotation group are

$$
J_{1}=-y_{3} p_{2}, \quad J_{2}=y_{3} p_{1}, \quad J_{3}=y_{1} p_{2}-y_{2} p_{1} .
$$


The Hamilton-Jacobi equation is

$$
H^{\prime}=\left(1-y_{1}^{2}\right) p_{1}^{2}-2 y_{1} y_{2} p_{1} p_{2}+\left(1-y_{2}^{2}\right) p_{2}^{2}+V\left(y_{1}, y_{2}\right)=E^{\prime}
$$

Now the Poisson bracket of functions $f\left(y_{1}, y_{2}, p_{1}, p_{2}\right), g\left(y_{1}, y_{2}, p_{1}, p_{2}\right)$ is

$$
\{f, g\}=\sum_{j=1}^{2}\left(\frac{\partial f}{\partial p_{j}} \frac{\partial g}{\partial y_{j}}-\frac{\partial f}{\partial y_{j}} \frac{\partial g}{\partial p_{j}}\right), \quad\left(y_{1}, y_{2}\right)=(x, y)
$$

We next determine the conditions that the function

$$
L=\sum_{j, k=1}^{2} a^{j k}\left(y_{1}, y_{2}\right) p_{j} p_{k}+W\left(y_{1}, y_{2}\right)=\ell+W, \quad a^{j k}=a^{k j}
$$

must satisfy to be a constant of the motion for $H^{\prime}$. It is straightforward to show that the requirement $\left\{H^{\prime}, L\right\}=0$ implies that the quadratic terms in $L$ are expressible as a second order polynomial in the $J_{k}$,

$$
\ell=\alpha_{1} J_{3}^{2}+\alpha_{2} J_{1}^{2}+\alpha_{3} J_{2}^{2}+\alpha_{4} J_{3} J_{1}+\alpha_{5} J_{3} J_{2}+\alpha_{6} J_{1} J_{2},
$$

and that (with $\partial_{y_{j}} W \equiv W_{j}$, etc.)

$$
\begin{aligned}
& W_{1}=\left(\frac{\left(1-y_{2}^{2}\right) a}{y_{3}^{2}}+\frac{y_{1} y_{2} b}{2 y_{3}^{2}}\right) V_{1}+\left(\frac{\left(1-y_{2}^{2}\right) b}{2 y_{3}^{2}}+\frac{y_{1} y_{2} c}{y_{3}^{2}}\right) V_{2} \\
& W_{2}=\left(\frac{y_{1} y_{2} a}{y_{3}^{2}}+\frac{\left(1-y_{1}^{2}\right) b}{2 y_{3}^{2}}\right) V_{1}+\left(\frac{y_{1} y_{2} b}{2 y_{3}^{2}}+\frac{\left(1-y_{1}^{2}\right) c}{y_{3}^{2}}\right) V_{2},
\end{aligned}
$$

where

$$
\begin{aligned}
& a\left(y_{1}, y_{2}\right)=\left(\alpha_{1}-\alpha_{3}\right) y_{2}^{2}+\alpha_{3}\left(1-y_{1}^{2}\right)-\alpha_{5} y_{2} y_{3} \\
& b\left(y_{1}, y_{2}\right)=-2 \alpha_{1} y_{1} y_{2}+\alpha_{4} y_{2} y_{3}+\alpha_{5} y_{1} y_{3}-\alpha_{6}\left(1-y_{1}^{2}-y_{2}^{2}\right) \\
& c\left(y_{1}, y_{2}\right)=\left(\alpha_{1}-\alpha_{2}\right) y_{1}^{2}+\alpha_{2}\left(1-y_{2}^{2}\right)-\alpha_{4} y_{1} y_{3} .
\end{aligned}
$$

The integrability conditions

$$
\partial_{x}\left(\partial_{y} W\right)=\partial_{y}\left(\partial_{x} W\right)
$$


are

$$
\begin{aligned}
& {\left[2\left(\alpha_{3}-\alpha_{1}\right) x y z+\alpha_{4} y\left(1-x^{2}\right)+\alpha_{5} x\left(1-x^{2}-2 y^{2}\right)+\alpha_{6} z\left(1-x^{2}\right)\right] } \\
\times & \left(V_{x x}-\frac{1-y^{2}}{x y} V_{x y}\right) \\
+ & {\left[2\left(\alpha_{1}-\alpha_{2}\right) x y z-\alpha_{4} y\left(1-2 x^{2}-y^{2}\right)-\alpha_{5} x\left(1-y^{2}\right)-\alpha_{6} z\left(1-y^{2}\right)\right] } \\
\times & \left(V_{y y}-\frac{1-x^{2}}{x y} V_{x y}\right) \\
= & \left(6\left[\alpha_{1}-\alpha_{3}\right] y z+3 \alpha_{4} x y-3 \alpha_{5}\left(1-x^{2}-2 y^{2}\right)+3 \alpha_{6} x z\right) V_{x} \\
+ & \left(6\left[\alpha_{2}-\alpha_{1}\right] x z+3 \alpha_{4}\left(1-2 x^{2}-y^{2}\right)-3 \alpha_{5} x y-3 \alpha_{6} y z\right) V_{y} .
\end{aligned}
$$

We denote the $V$ solution space of this equation by

$$
\left[\alpha_{1}, \cdots, \alpha_{6}\right]
$$

Let us now return to our assumption that the Hamilton-Jacobi equation admits two constants of the motion:

$$
L_{h}=\sum_{j, k=1}^{2} a_{(h)}^{j k} p_{k} p_{j}+W_{(h)}, \quad h=1,2 .
$$

These two operators together with $H^{\prime}$ are assumed functionally independent. The constant of the motion $L_{1}$ leads to the condition (21) on the potential $V$; whereas $L_{2}$ leads to the second condition

$$
\left[\beta_{1}, \cdots, \beta_{6}\right]
$$

The potential must lie in the intersection of the solution spaces $(21,22)$ for these two conditions. It follows that the equations

$$
V_{x x}-\frac{1-y^{2}}{x y} V_{x y}=A V_{x}+B V_{y}, \quad V_{y y}-\frac{1-x^{2}}{x y} V_{x y}=C V_{x}+D V_{y}
$$

must hold, where

$$
\begin{aligned}
A \mathcal{E} & =-12 H_{12} x y^{2} z^{2}+6 H_{14} y^{2} z\left(y^{2}-1+x^{2}\right)+12 H_{13} x y^{2} z^{2}+6 H_{16} y z^{2}\left(y-x^{2}-1\right) \\
& -6 H_{15} x y z\left(x^{2}+y^{2}\right)+6 H_{25} x y z\left(2 y^{2}+x^{2}-1\right)+6 H_{26} x^{2} y z^{2}-12 H_{23} x y^{2} z^{2}
\end{aligned}
$$




$$
\begin{aligned}
& +6 H_{24} x^{2} y^{2} z+6 H_{34} y^{2} z\left(1-2 x^{2}-y^{2}\right)+6 H_{36} y z^{2}\left(1-y^{2}\right)+6 H_{35} x y z\left(1-y^{2}\right) \\
& +3 H_{45} y\left(2 x^{2}-1-2 y^{4}-4 x^{2} y^{2}+3 y^{2}-2 x^{4}\right)-6 H_{46} x^{3} y z+3 H_{56} z\left(2 y^{4}-3 y^{2}+1\right) \\
B \mathcal{E} & =6 H_{15} x^{2} z+12 H_{14} x y z\left(1-y^{2}-2 x^{2}\right)+6 H_{16} x z^{2}-6 H_{26} x z^{2} \\
& +12 H_{24} x y z\left(2 x^{2}+y^{2}-1\right)-6 H_{25} x^{2} z+3 H_{46} z\left(1-2 x^{2}-y^{2}\right)\left(1-2 y^{2}\right) \\
& +3 H_{45} x\left(1-2 x^{2}-y^{2}\right)\left(1-2 y^{2}\right) \\
2 C \mathcal{E} & =-6 H_{16} y z^{2}-6 H_{14} y^{2} z+6 H_{36} y z^{2}+6 H_{34} y^{2} z \\
& +3 H_{45} y\left(2 y^{2}+x^{2}-1\right)+3 H_{56} z\left(1-2 y^{2}-x^{2}\right) \\
2 D \mathcal{E} & =-12 H_{12} x^{2} y z^{2}+6 H_{16} x z^{2}\left(1-x^{2}+y^{2}\right)+6 H_{15} x^{2} z\left(1-y^{2}-x^{2}\right) \\
& +12 H_{13} x^{2} y z^{2}+6 H_{14} x y z\left(2-3 x^{2}-y^{2}\right)+6 H_{24} x y z\left(x^{2}-1\right) \\
& -6 H_{26} x z^{2}\left(1-x^{2}\right)+6 H_{25} x^{2} z\left(2 y^{2}+x^{2}-1\right)-12 H_{23} x^{2} y z^{2} \\
& +6 H_{34} x y z\left(2 x^{2}+y^{2}-1\right)-6 H_{36} x y^{2} z^{2}-6 H_{35} x^{2} y^{2} z \\
& +3 H_{45} x\left(1-4 y^{2}+6 x^{2} y^{2}-3 x^{2}+2 x^{4}+2 y^{4}\right)+3 H_{46} z\left(1-x^{2}\right)\left(1-2 x^{2}-2 y^{2}\right) \\
& +6 H_{56} x y^{3} z \\
\mathcal{E} & =-4 H_{13} x^{2} y^{2}\left(1-x^{2}-y^{2}\right)+2 H_{16}\left(1-x^{2}\right)\left(x^{2}-y^{2}\right) x y+2 H_{15} x^{2} y z\left(y^{2}+x^{2}\right) \\
& +4 H_{12} x^{2} y^{2}\left(1-x^{2}-y^{2}\right)-2 H_{14} x y^{2} z\left(x^{2}+y^{2}\right)-2 H_{26} x y\left(1-x^{2}-y^{2}\right)\left(x^{2}-1\right) \\
& +2 H_{24} x y^{2} z\left(1-x^{2}\right)-2 H_{25} x^{2} y z\left(2 y+x^{2}-1\right)+4 H_{23} x^{2} y^{2}\left(1-x^{2}-y^{2}\right) \\
& +2 H_{36} x y\left(1-x^{2}-y^{2}\right)\left(y^{2}-1\right)-2 H_{34} x y^{2} z\left(1-2 x^{2}-y^{2}\right)+2 H_{35} x^{2} y z\left(y^{2}-1\right) \\
& -2 H_{45} x y\left(y^{2}-y^{4}-2 x^{2} y^{2}-x^{4}+x^{2}\right)-2 H_{46} x^{2} y z\left(1-x^{2}\right)-2 H_{56} x y^{2} z\left(y^{2}-1\right),
\end{aligned}
$$

and $H_{k \ell}=-H_{\ell k}=\alpha_{k} \beta_{\ell}-\alpha_{\ell} \beta_{k}$.

Differentiating each of equations (23) with respect to $x$ and $y$, we obtain 4 equations for the 4 third derivatives of $V$, expressed in terms of $V_{x}, V_{y}, V_{x y}$ :

$$
\begin{gathered}
V_{x x x}=\frac{1}{x y\left(-1+y^{2}+x^{2}\right)}\left(\left(x y^{3}-x y+y x^{3}\right) B_{x}+\left(y x-2 y^{3} x\right) \mathcal{E}_{x}+\left(y x^{3}-y x+y^{3} x\right) A B\right. \\
+\left(y^{2} x^{2}-y^{4} x^{2}\right) B_{y}+\left(y x+y^{5} x\right) C B+y^{5} x \mathcal{E}_{x} \\
\left.-2 y^{3} x C B+\left(y^{2} x^{2}-y^{4} x^{2}\right) B \mathcal{E}\right) V_{y} \\
+\frac{1}{y x\left(-1+y^{2}+x^{2}\right)}\left(\left(x^{3} y-x y+x y^{3}\right) A_{x}+\left(y^{2} x^{2}-y^{4} x^{2}\right) A_{y}\right. \\
+\left(y x+y^{5} x-2 y^{3} x\right) C_{x}+\left(y x+y^{5} x-2 y^{3} x\right) C A+\left(y^{2} x^{2}-y^{4} x^{2}\right) B C
\end{gathered}
$$




$$
\begin{aligned}
& \left.+\left(y^{3} x+y x^{3}-y x\right) A^{2}\right) V_{x} \\
& +\frac{1}{y x\left(-1+y^{2}+x^{2}\right)}\left(\left(2 y^{2}+x^{2}-y^{4}-y^{4} x^{2}-1\right) A+\left(3 y^{4}-y^{6}-3 y^{2}\right) C+y^{3} x^{3} B+y x \mathcal{E}\right. \\
& \left.-3 x-2 y^{3} x \mathcal{E}+3 x y^{2}+y^{5} x \mathcal{E}\right) V_{x y} \\
& V_{x x y}=\frac{-1}{x\left(-1+y^{2}+x^{2}\right)}\left(-x^{3} y^{2} B E+y^{3} x^{2} \mathcal{E}_{x}-x^{3} y^{2} B_{y}\right. \\
& \left.-x^{2} y C B-x^{2} * y \mathcal{E}_{x}+y^{3} x^{2} C B\right) V_{y} \\
& -\frac{1}{x\left(-1+y^{2}+x^{2}\right)}\left(-x^{3} y^{2} A_{y}+y^{3} x^{2} C_{x}-x^{2} y C A+y^{3} x^{2} C A\right. \\
& \left.-x^{2} y C_{x}-x^{3} y^{2} B C\right) V_{x} \\
& -\frac{1}{x\left(-1+y^{2}+x^{2}\right)}\left(-x^{2} y B-x^{3} y^{2} A-y^{2}-x^{2} y \mathcal{E}+1+y^{3} x^{2} \mathcal{E}\right. \\
& \left.-x y^{4} C-x C+2 x^{2}+x^{4} y B+2 x y^{2} C\right) V_{x y} \\
& V_{x y y}=\frac{1}{y\left(-1+y^{2}+x^{2}\right)}\left(-x^{3} y^{2} B \mathcal{E}+\left(x y^{2}-x^{3} y^{2}\right) B_{y}+y^{2} x B \mathcal{E}\right. \\
& \left.+y^{3} x^{2} \mathcal{E}_{x}+y^{3} x^{2} C B\right) V_{y} \\
& +\frac{1}{y\left(-1+y^{2}+x^{2}\right)}\left(\left(x y^{2}-x^{3} y^{2}\right) A_{y}+\left(y^{2} x-x^{3} y^{2}\right) B C\right. \\
& \left.+y^{3} x^{2} C A+y^{3} x^{2} C_{x}\right) V_{x} \\
& +\frac{1}{y\left(-1+y^{2}+x^{2}\right)}\left(\left(x y^{2}-x^{3} y^{2}\right) A+\left(x^{4} y-2 x^{2} y+y\right) B+x^{2}+y^{3} x^{2} \mathcal{E}\right. \\
& \left.-\left(x y^{4}+x y^{2}\right) C(x, y)-2 y^{2}-1\right) V_{x y} \\
& V_{y y y}=\frac{1}{y x\left(-1+y^{2}+x^{2}\right)}\left(\left(x^{3} y+x y^{3}-x y\right) \mathcal{E}_{y}+\left(y^{2} x^{2}-x^{4} y^{2}\right) \mathcal{E}_{x}-x^{4} y^{2} C B\right. \\
& +y x B \mathcal{E}+x^{5} y B_{y}+y x^{3} \mathcal{E}^{2}+\left(x y-2 x^{3} y\right) B_{y}-2 x^{3} y B \mathcal{E}
\end{aligned}
$$




$$
\begin{gathered}
\left.+y^{2} x^{2} B C+x^{5} y B \mathcal{E}+\left(y^{3} x-y x\right) \mathcal{E}^{2}\right) V_{y} \\
+\frac{1}{y x\left(-1+y^{2}+x^{2}\right)}\left(\left(y^{2} x^{2}-x^{4} y^{2}\right) C_{x}+\left(x y+x^{5} y-2 x^{3} y\right) A_{y}\right. \\
+\left(x^{3} y-x y+x y^{3}\right) C_{y}+\left(y^{3} x-y x+x^{3} y\right) \mathcal{E} C+\left(y^{2} x^{2}-x^{4} y^{2}\right) C A \\
\left.+\left(x y-2 x^{3} y+x^{5} y\right) B C\right) V_{x} \\
+\frac{1}{y x\left(-1+y^{2}+x^{2}\right)}\left(\left(y^{2}-x^{4} y^{2}+2 x^{2}-x^{4}-1\right) \mathcal{E}+\left(x^{5} y+x y-2 x^{3} y\right) A+x^{3} y^{3} C\right. \\
\left.+\left(3 x^{4}-x^{6}-3 x^{2}+1\right) B-3 y+3 x^{2} y\right) V_{x y} .
\end{gathered}
$$

Thus if the potential $V$ is subject to the two conditions $(21,22)$, then $V$ can depend on at most 3 parameters, in addition to a trivial additive constant. We can choose these parameters to be $V_{x}\left(x_{0}, y_{0}\right), V_{y}\left(x_{0}, y_{0}\right), V_{x y}\left(x_{0}, y_{0}\right)$ for any fixed regular point $\left(x_{0}, y_{0}\right)$. Then $V_{x x}\left(x_{0}, y_{0}\right), V_{y y}\left(x_{0}, y_{0}\right)$ and all higher derivatives can be computed in terms of $V_{x}, V_{y}, V_{x y}$ by successive differentiation and utilisation of relations (23).

We require that our potential be nondegenerate, i.e., that it depend on 3 arbitrary parameters. Then, the 3 conditions $\partial_{y} V_{x x x}=\partial_{x} V_{x x y}, \partial_{y} V_{x x y}=$ $\partial_{x} V_{x y y}$ and $\partial_{y} V_{x y y}=\partial_{x} V_{y y y}$ for the fourth partial derivatives lead to 9 integrability conditions, since we can equate the coefficients of $V_{x}, V_{y}$, and $V_{x y}$ in each of these identities. (Otherwise $V$ would necessarily depend on less than 3 arbitrary parameters.)

Note that if we have another constant of the motion $L_{3}$ associated with a nondegenerate potential, then $L_{3}$ must be a linear combination of $H^{\prime}=$ $J_{1}^{2}+J_{2}^{2}+J_{3}^{2}, L_{1}, L_{2}$. Indeed, if $L_{3}$ is not a linear combination of the basis functions, then the potential $V$ must satisfy an equation (10) that is linearly independent of the equations associated with $L_{1}, L_{2}$. This means an additional constraint on the solution space and that $V$ can depend on at most two parameters, which is a contradiction.

The integrability conditions are only guaranteed to be necessary conditions for the existence of a 3 parameter potential. However, we shall find that they are in fact sufficient. Each of the 9 conditions can be expressed as a polynomial identity in the variables $x, y$ whose coefficients are homogenious polynomials in the coefficients $H_{i j}$. Since these relations must hold identically in $x, y$ we can equate to zero each of the components in the polynomial 
expansion. The resulting expressions are lengthy; we used the symbol manipulation program MAPLE to compute them. Even then they would have been very cumbersome to solve if we had not been able to simplify the computation further by taking advantage of $S O(3, C)$ equivalence.

We will now use the 9 conditions to classify the possible potentials $V$ and the corresponding constants of the motion $L_{1}, L_{2}$. For this we note that it is only the three-dimensional subspace spanned by $H, L_{1}, L_{2}$ that matters; we can choose any basis for this subspace. Hence we can replace the conditions $(21,22)$ by linear combinations of themselves without changing the potential. Moreover, to further simplify the results we note that we can always subject the coordinates $(x, y)$, and $L_{1}, L_{2}$ to a simultaneous complex rotation motion, i.e., we regard all translated and rotated potentials as members of the same equivalence class.

We will consider two superintegrable systems on the complex sphere as the same if one system can be transformed to the other via an action of the complex orthogonal group $S O(3, C)$. One can identify the adjoint action of $S O(3, C)$ on the second order elements in the enveloping algebra of $s o(3, C)$ with the action via similarity transformation of this group on the space of $3 \times 3$ complex symmetric matrices. A straight-forward computation shows that this actions divides the second order elements into orbits. A representative from each orbit class is given by:

$$
\begin{aligned}
& {[1] \quad J_{3}^{2},} \\
& {[2] \quad\left(J_{1}+i J_{2}\right)^{2},} \\
& {[3] \quad J_{3}\left(J_{1}+i J_{2}\right),} \\
& {[4] \quad J_{1}^{2}+r^{2} J_{2}^{2}, \quad\left|r^{2}\right| \leq 1, r^{2} \neq 0,1,} \\
& {[5] \quad\left(J_{1}+i J_{2}\right)^{2}+s J_{3}^{2}, \quad s \neq 0,} \\
& {[6] \quad J \cdot J=J_{1}^{2}+J_{2}^{2}+J_{3}^{2} .}
\end{aligned}
$$

Here, $J \cdot J$ is invariant under the group action, and we can add arbitrary multiples of $J \cdot J$ to any of these operators without changing the orbit class.

Our strategy to classify the three-dimensional subspaces of operators corresponding to maximal parameter dependent potentials is as follows. We choose $\ell_{1}$ from one of the orbit classes (29)-(33), where $L_{1}=\ell_{1}+W_{1}$. We first take take $\ell_{2},\left(L_{2}=\ell_{2}+W_{2}\right)$ as a general operator $\left[\beta_{1}, \cdots, \beta_{6}\right]$. However, we can then simplify $\ell_{2}$ by adding arbitrary multiples of $\ell_{1}$ and $J \cdot J$ 
to it, by multiplying $\ell_{2}$ by any nonzero complex number, and by applying a complex rotation to $\ell_{2}$ that leaves $\ell_{1}$ invariant. Note that $J \cdot J$ is invariant under all complex rotations. Finally we apply the 9 integrability conditions to $L_{1}$ and the simplified $L_{2}$, to determine those choices of $L_{2}$ that admit the nondegenerate potentials.

Suppose $\ell_{1}=J_{3}^{2}$. This operator is invariant under an arbitrary complex rotation about the z-axis. Such a rotation will leave $\beta_{1}, I_{1}=\beta_{2}+\beta_{3}, I_{2}=$ $\beta_{2} \beta_{3}-\beta_{6}^{2} / 4$ and $I_{3}=\beta_{4}^{2}+\beta_{5}^{2}$ invariant. If we can rotate such that the transformed $\beta_{6}=0$ then we can achieve the form $\left[0,1,0, \beta_{4}, \beta_{5}, 0\right]$. The integrability conditions require $\beta_{4}=\beta_{5}=0$.

Case (1a)

$$
[1,0,0,0,0,0], \quad[0,1,0,0,0,0]
$$

Here,

$$
\begin{aligned}
L_{1} & =J_{3}^{2}+W^{(1)}, \quad L_{2}=J_{1}^{2}+W^{(2)} \\
V(x) & =\frac{\alpha}{x^{2}}+\frac{\beta}{y^{2}}+\frac{\gamma}{1-x^{2}-y^{2}} .
\end{aligned}
$$

This potential allows separation in spherical and ellipsoidal coordinates.

If $I_{1}=I_{2}=0$ but not all of $\beta_{2}, \beta_{3}, \beta_{6}$ are zero, then we can rotate to achieve $\left[0,1,-1, \beta_{4}, \beta_{5}, 2 i\right]$. Again integrability conditions require $\beta_{4}=\beta_{5}=$ 0 .

\section{Case (1b)}

$$
[1,0,0,0,0,0], \quad[0,1,-1,0,0,2 i]
$$

Here,

$$
\begin{aligned}
L_{1} & =J_{3}^{2}+W^{(1)}, \quad L_{2}=\left(J_{1}+i J_{2}\right)^{2}+W^{(2)} \\
V(x) & =\frac{\alpha}{z^{2}}+\frac{\beta}{(x+i y)^{2}}+\frac{\gamma(x-i y)}{(x+i y)^{3}}
\end{aligned}
$$

This potential allows separation in spherical, horospherical and degenerate elliptical coordinates of type 1 . 
If $\beta_{2}=\beta_{3}=\beta_{6}=0, I_{3}=0$, we can achieve $\beta_{5}=-i \beta_{4}$, which satisfies the integrability conditions.

\section{Case (1c)}

$$
[1,0,0,0,0,0], \quad[0,0,0,1,-i, 0]
$$

Here,

$$
\begin{aligned}
L_{1} & =J_{3}^{2}+W^{(1)}, \quad L_{2}=J_{3}\left(J_{1}+i J_{2}\right)+W^{(2)} \\
V(x) & =\frac{\alpha}{(x+i y)^{2}}+\beta \sqrt{\frac{1-x^{2}-y^{2}}{x^{2}+y^{2}}}+\frac{i \gamma}{\sqrt{(x-i y)(x+i y)^{2}}} .
\end{aligned}
$$

Separability is possible in spherical coordinates and degenerate elliptic coordinates of type 2 .

If $I_{3} \neq 0$ we have

Case (1d)

$$
[1,0,0,0,0,0], \quad[0,0,0,1,0,0]
$$

Here,

$$
\begin{aligned}
L_{1} & =J_{3}^{2}+W^{(1)}, \quad L_{2}=J_{3} J_{1}+W^{(2)} \\
V(x) & =\frac{\alpha z}{\sqrt{x^{2}+y^{2}}}+\frac{1}{\sqrt{x^{2}+y^{2}}}\left[\frac{\beta}{\sqrt{x^{2}+y^{2}}+x}+\frac{\gamma}{\sqrt{x^{2}+y^{2}}-x}\right] .
\end{aligned}
$$

Separation of variables is possible in spherical coordinates and rotated ellipsoidal coordinates, $\left\{R_{1}, R_{2}\right\}$. A suitable choice of the latter is

$$
\begin{gathered}
z_{1}=\frac{i}{2} \frac{\left(A_{+}^{2}+A_{-}^{2}\right)\left(R_{1} R_{2}+1\right)+2 A_{+} A_{-}\left(R_{1}+R_{2}\right)}{\left(A_{+}^{2}-A_{-}^{2}\right) \sqrt{R_{1} R_{2}}}, \quad z_{3}=-\frac{1}{2} \frac{R_{1} R_{2}+1}{\sqrt{R_{1} R_{2}}}, \\
z_{2}=\frac{\sqrt{\left(A_{+} R_{1}+A_{-}\right)\left(A_{-} R_{1}+A_{+}\right)\left(A_{+} R_{2}+A_{-}\right)\left(A_{-} R_{2}+A_{+}\right)}}{\left(A_{+}^{2}-A_{-}^{2}\right) \sqrt{R_{1} R_{2}}},
\end{gathered}
$$

with the associated operator $\left(A_{-}^{2}-A_{+}^{2}\right) J_{1} J_{3}-i\left(A_{+}^{2}+A_{-}^{2}\right) J_{3}^{2}$. 
Now suppose $\ell_{1}=J_{1}^{2}+r^{2} J_{2}^{2}$, corresponding to (32). In this case there is no simplification possible by rotation and we must apply the integrability conditions for general $\left[0,0, \beta_{3}, \beta_{4}, \beta_{5}, \beta_{6}\right]$. We find that the integrability conditions are satisfied if and only if $\beta_{3}= \pm i r, \beta_{3}=\beta_{4}=0$ and $\beta_{6}=1$.

Case (2)

$$
\left[0,1, r^{2}, 0,0,0\right], \quad[0,0, i r, 0,0,1]
$$

Here,

$$
\begin{aligned}
L_{1} & =\left(c^{2}-1\right)^{2} J_{3}^{2}-4 c^{2} J_{1}^{2}+W^{(1)}, \quad L_{2}=c^{2} J_{3}^{2}-\left(J_{1}-i J_{2}\right)^{2}+W^{(2)} \\
V(x) & =\frac{\alpha\left(z_{+}+c^{2} z_{-}\right)}{\sqrt{\left(c^{2} z_{-}-z_{+}\right)^{2}-4 c^{2} z_{3}^{2}}}+\frac{\beta\left(z_{+}-c^{2} z_{-}\right)\left(z_{+} z_{-}+z_{3}^{2}\right)}{z_{3}^{2} \sqrt{\left(c^{2} z_{-}-z_{+}\right)^{2}-4 c^{2} z_{3}^{2}}}+\frac{\gamma z_{+} z_{-}}{z_{3}^{2}},(48)
\end{aligned}
$$

$z_{ \pm}=x \pm i y, z_{3}=\sqrt{1-x^{2}-y^{2}}$ and $c^{2}=(1+r) /(1-r)$. Separation of variables is possible in elliptical and elliptic parabolic coordinates.

Next suppose $\ell_{1}=\left(J_{1}-i J_{2}\right)^{2}$. Then, eliminating Case $1 \mathrm{~b}$ above, the only posibilities for $L_{2}$ are operators of the form $\left[\beta_{1}, 1,0 \beta_{4}, \beta_{5}, 0\right]$, or $\left[\beta_{1}, 0,0,1,0,0\right]$, or $\left[\beta_{1}, 0,0,1,-i, 0\right]$, and only the last of these with $\beta_{1}=0$ satisfies the integrability conditions.

Case (3)

$$
[0,1,-1,0,0,-2 i], \quad[0,0,0,1,-i, 0]
$$

where,

$$
\begin{aligned}
L_{1} & =\left(J_{1}-i J_{2}\right)^{2}+W^{(1)}, \quad L_{2}=J_{3}\left(J_{1}-i J_{2}\right)+W^{(2)} \\
V(x) & =\frac{\alpha}{(x+i y)^{2}}+\frac{\beta z}{(x+i y)^{3}}+\frac{\gamma\left(1-4 z^{2}\right)}{(x+i y)^{4}} .
\end{aligned}
$$

Separation of variables is possible in spherical coordinates and rotated semicircular parabolic coordinates.

One can verify from the integrability conditions that orbit (33) does not occur for any nondegenerate potential. This completes the classification of these potentials.

For a general choice of operators $L_{1}, L_{2}$ it is not the case that $R^{2}$ is a polynomial in $L_{0}, L_{1}, L_{2}$, i.e., there is no quadratic algebra structure. However, we can demonstrate that there is a quadratic algebra associated with each 
nondegenerate potential above. Because we are working in two dimensions there can only be three functionally independant constants at most. Consequently all Poisson brackets must be functionally dependent on $H=L_{0}, L_{1}$ and $L_{2}$. We want to show that in fact $R^{2}=\left\{L_{1}, L_{2}\right\}^{2}=F\left(L_{0}, L_{1}, L_{2}\right)$ is a polynomial in these variables. First, we can verify that this is true when the potential is turned off, i.e., if we consider only the functions

$$
\ell_{h}=\sum_{j, k=1}^{2} a_{(h)}^{j k} p_{k} p_{j}, h=1,2 \quad \ell_{0}=\left(1-y_{1}^{2}\right) p_{1}^{2}-2 y_{1} y_{2} p_{1} p_{2}+\left(1-y_{2}^{2}\right) p_{2}^{2}
$$

where $L_{h}=\ell_{h}+W^{(h)}$. Let $\mathcal{R}=\left\{\ell_{1}, \ell_{2}\right\}$. Then for each of the cases $1-3$ listed above it is straightforward to check that $\mathcal{R}^{2}=\mathcal{P}_{3}\left(\ell_{0}, \ell_{1}, \ell_{2}\right)$ where $\mathcal{P}_{3}$ is a homogeneous third order polynomial in its arguments. ${ }^{1}$ It follows that

$$
R^{2}=F\left(L_{0}, L_{1}, L_{2}\right)=\mathcal{P}_{3}\left(L_{0}, L_{1}, L_{2}\right)+F_{4}\left(\mathbf{s}, L_{0}, L_{1}, L_{2}\right),
$$

where $F_{4}$ is a fourth, second and zeroth order polynomial in the momenta $p_{x}, p_{y}$, and $F_{4}\left(\mathbf{0}, L_{0}, L_{1}, L_{2}\right)=0$. Here, the parameters in the potential are denoted by $\mathbf{s}=\left(V_{x}^{0}, V_{y}^{0}, V_{y y}^{0}\right)$, evaluated at some fixed point $\left(x_{0}, y_{0}\right)$ and $F_{4}$ is a polynomial function of these parameters.

From the definition of the Poisson bracket we have

$$
\begin{gathered}
\left\{\ell_{1}, \mathcal{R}\right\}=\frac{1}{2} \frac{\partial \mathcal{P}_{3}}{\partial \ell_{2}}\left(\ell_{0}, \ell_{1}, \ell_{2}\right), \\
\left\{\ell_{2}, \mathcal{R}\right\}=-\frac{1}{2} \frac{\partial \mathcal{P}_{3}}{\partial \ell_{1}}\left(\ell_{0}, \ell_{1}, \ell_{2}\right)
\end{gathered}
$$

hence

$$
\begin{gathered}
\left\{L_{1}, R\right\}=\frac{1}{2} \frac{\partial \mathcal{P}_{3}}{\partial L_{2}}\left(L_{0}, L_{1}, L_{2}\right)+\frac{1}{2} \frac{\partial F_{4}}{\partial L_{2}}(\mathbf{s}), \\
\left\{L_{2}, R\right\}=-\frac{1}{2} \frac{\partial \mathcal{P}_{3}}{\partial L_{1}}\left(L_{0}, L_{1}, L_{2}\right)-\frac{1}{2} \frac{\partial F_{4}}{\partial L_{1}}(\mathbf{s}),
\end{gathered}
$$

\footnotetext{
${ }^{1}$ Moreover, it is straightforward to verify that the cases corresponding to nondegenerate potentials are the only cases where $\mathcal{P}_{3}$ is a homogeneous third order polynomial in its arguments. Thus the possible quadratic algebras generated by second order elements in the Lie algebra of $S O(3, C)$ correspond one-to-one with nondegenerate potentials.
} 
where the $\partial F_{4} / \partial L_{h}(\mathbf{s})$ have only terms of orders two and zero in the momenta. It follows that the $\partial F_{4} / \partial L_{h}(\mathbf{s})$ must be expressible as linear combinations of the $L_{h}$. This shows that the commutators $\left\{L_{h}, R\right\}$ can be expressed as polynomials in $L_{0}, L_{1}, L_{2}$. It is then a simple matter to verify that $F$ itself is a polynomial in $L_{0}, L_{1}, L_{2}$.

We now list the quadratic algebra relations for each of the cases studied above. In view of relations

$$
\left\{L_{1}, R\right\}=\frac{1}{2} \frac{\partial F}{\partial L_{2}}, \quad\left\{L_{2}, R\right\}=-\frac{1}{2} \frac{\partial F}{\partial L_{1}} .
$$

it is sufficient to give the relation $R^{2}=F\left(L_{0}, L_{1}, L_{2}\right)$ for each case.

Case (1a) $\quad[1,0,0,0,0,0], \quad[0,1,0,0,0,0]$

$R^{2}=16 L_{2} L_{1}\left(H^{\prime}-L_{1}-L_{2}-\alpha-\beta-\gamma\right)-16\left[\alpha L_{2}^{2}+\beta\left(H^{\prime}-L_{1}-L_{2}-\alpha-\beta-\gamma\right)^{2}+\gamma L_{1}^{2}\right]$

$$
+64 \alpha \beta \gamma \text {. }
$$

Case (1b) $\quad[1,0,0,0,0,0], \quad[0,1,-1,0,0,2 i]$

$$
\begin{gathered}
R^{2}=-16 L_{2}^{2} L_{1}-16 \gamma{H^{\prime 2}}^{2}-16 \gamma L_{1}^{2}-16 \beta H^{\prime} L_{2}-32 \gamma H L_{1}-16 \beta L_{2} L_{1}+16 \alpha \beta L_{2} \\
-32 \alpha \gamma L_{1}+32 \alpha \gamma H^{\prime}+16 \alpha \gamma(\gamma-\alpha) .
\end{gathered}
$$

Case (1c) $\quad[1,0,0,0,0,0], \quad[0,0,0,1,-i, 0]$

$$
R^{2}=-4 L_{2}^{2} L_{1}-4 \alpha L_{1}^{2}+4 \alpha H^{\prime} L_{1}+2 \gamma L_{2} L_{1}+i \beta \gamma L_{2}-\frac{\gamma^{2}}{4} H^{\prime}+\beta^{2} \alpha-\frac{i \beta^{2}}{4} .
$$

Case (1d) $\quad[1,0,0,0,0,0], \quad[0,0,0,1,0,0]$

$$
\begin{gathered}
R^{2}=-4 L_{1}^{3}-4 L_{2}^{2} L_{1}+4 L_{1}^{2} H^{\prime}-2(\beta+\gamma) L_{1}^{2}-2(\beta+\gamma) L_{2}^{2}+4 \alpha L_{1} L_{2}+4 \beta \gamma L_{1}+4 \alpha \beta L_{2} \\
-4 \beta \gamma H^{\prime}+2 \beta \gamma(\beta+\gamma)-2 \beta \alpha^{2} .
\end{gathered}
$$

Case (2) $\quad\left[0,1, r^{2}, 0,0,0\right], \quad[0,0, i r, 0,0,1]$

$$
\begin{gathered}
R^{2}=32 c^{4}\left(c^{2}-1\right) H^{\prime 3}-16 c^{2}\left(c^{4}-1\right) L_{2}^{3}-16 c^{2} L_{1}^{2} L_{2}+32 c^{2}\left(c^{2}-1\right)\left(2 c^{2}+1\right) L_{2}^{2} H^{\prime} \\
+16\left(2 c^{2}-1\right) L_{2}^{2} L_{1}-16\left(c^{2}-1\right)\left(5 c^{2}+1\right) c^{2} H^{\prime 2} L_{2}+16 c^{4} H^{\prime 2} L_{1} \\
-32 c^{2}\left(2 c^{2}-1\right) L_{2} L_{1} H^{\prime}+64 \gamma\left(c^{4}-1\right) L_{2}^{2}-128 \gamma c^{2}\left(c^{2}-1\right) H^{\prime} L_{2}-64 \gamma c^{2} L_{2} L_{1} \\
+16 c^{2}\left[\left(c^{2}(\alpha-\beta)\right)^{2}-(\alpha+\beta)^{2}\right] L_{2}-16 c^{4}(\beta-\alpha)^{2} L_{1}-32 c^{4}(\beta-\alpha)\left(c^{2}(\beta-\alpha)+\beta+\alpha\right) H^{\prime} \\
-64 c^{4} \gamma(\beta-\alpha)^{2} .
\end{gathered}
$$

Case (3) $\quad[0,1,-1,0,0,2 i], \quad[0,0,0,1, i, 0]$

$$
R^{2}=4 L_{1}^{3}+16 \gamma L_{2}^{2}+8 \alpha L_{1}^{2}+16 \gamma H^{\prime} L_{1}-4 \beta L_{2} L_{1}-4 \beta \alpha L_{2}-\beta^{2} H^{\prime} .
$$




\section{Quantum superintegrability on the two di- mensional sphere}

Here we give the analogous quantum algebras for superintegrable systems arising from the potentials we have already computed. The main difference is that the Poisson bracket is now replaced by the commutator bracket $[A, B]=$ $A B-B A$ and the operators $H, L_{1}$ and $L_{2}$ are the obvious (formally selfadjoint) symmetry partial differential operators, built from the symmetry operators

$$
J_{1}=-z \partial_{y}, \quad J_{2}=z \partial_{x}, \quad J_{3}=x \partial_{y}-y \partial_{x},
$$

where $z=\sqrt{1-x^{2}-y^{2}}$ and

$$
H=J_{1}^{2}+J_{2}^{2}+J_{3}^{2}+V(x, y), \quad L_{h}=\sum_{k, j=1}^{2} \partial_{k}\left(a_{(h)}^{k j}\right) \partial_{j}+W_{(h)}(x, y), \quad h=1,2
$$

The Hamilton-Jacobi equation is replaced by the Schrödinger equation

$$
H \Psi=E \Psi
$$

Just as for the Hamilton-Jacobi case, if we have another constant of the motion (symmetry operator) $L_{3}$ associated with a maximal potential, then $L_{3}$ must be a linear combination of $H, L_{1}, L_{2}$. Indeed, if $L_{3}$ is in self-adjoint form, then the conditions that $\left[H, L_{3}\right]=0$ are identical with (16), (where we replace $J_{h} J_{k}$ by $\left.\frac{1}{2}\left\{J_{h}, J_{k}\right\}\right)$ and $(17,18)$. Thus, if $L_{3}$ is not a linear combination of the basis functions, then the potential $V$ must satisfy an equation (10) that is linearly independent of the equations associated with $L_{1}, L_{2}$. This means an additional constraint on the solution space and that $V$ can depend on at most two parameters, which is a contradiction.

Furthermore the proof of the existence of quadratic algebra relations at the end of $\S 2$ goes through almost unchanged for the operator case: $\left[L_{1}, L_{2}\right]^{2}=R^{2}$ and $\left[L_{1}, R\right],\left[L_{2}, R\right]$ can be expressed as (symmetric) polynomials in the operators $L_{1}, L_{2}, H$. To make the prior construction go through, one need only note that since $R^{2}$ is a formally self-adjoint 6th order differential symmetry operator, the 5th order terms are fixed linear functions of the 6 th order terms. The expressions $\{A, B\}=A B+B A$ and $\{A, B, C\}=$ $A B C+C A B+B C A$ are operator symmetrizers. The explicit relations are: 


\section{Case (1a)}

$$
\begin{aligned}
{\left[L_{i}, R\right] } & =4\left\{L_{i}, L_{k}\right\}-4\left\{L_{i}, L_{j}\right\}-\left(8+16 a_{j}\right) L_{j}+\left(8+16 a_{k}\right) L_{k}+8\left(a_{j}-a_{k}\right) \\
R^{2} & =\frac{8}{3}\left\{L_{1}, L_{2}, L_{3}\right\}-\left(16 a_{1}+12\right) L_{1}^{2}-\left(16 a_{2}+12\right) L_{2}^{2}-\left(16 a_{3}+12\right) L_{3}^{2} \\
& +\frac{52}{3}\left(\left\{L_{1}, L_{2}\right\}+\left\{L_{1}, L_{3}\right\}+\left\{L_{2}, L_{3}\right\}\right)+\frac{1}{3}\left(16+176 a_{1}\right) L_{1} \\
& +\frac{1}{3}\left(16+176 a_{2}\right) L_{2}+\frac{1}{3}\left(16+176 a_{3}\right) L_{3}+\frac{32}{3}\left(a_{1}+a_{2}+a_{3}\right) \\
& +48\left(a_{1} a_{2}+a_{1} a_{3}+a_{2} a_{3}\right)+64 a_{1} a_{2} a_{3}
\end{aligned}
$$

here, $a_{1}=\gamma, a_{2}=\alpha, a_{3}=\beta, L_{1}+L_{2}+L_{3}+\sum a_{h}=H$ and $i, j, k$ are chosen such that $\epsilon_{i j k}=1$, where $\epsilon$ is the purely skew-symmetric tensor.

Case (1b)

$$
\begin{aligned}
{\left[R, L_{2}\right] } & =8 L_{2}^{2}+16 \gamma H+8 \beta L_{2}+16 \gamma L_{1}-(8 \gamma-16 \alpha \gamma) \\
{\left[R, L_{1}\right] } & =-8\left\{L_{2}, L_{1}\right\}-8 \beta H-16 L_{2}-8 \beta L_{1}+8 \beta(\alpha-1) \\
R^{2} & =-\frac{8}{3}\left\{L_{2}, L_{2}, L_{1}\right\}-16 \gamma H^{2}-\frac{176}{3} L_{2}^{2}-16 \gamma L_{1}^{2}-16 \beta H L_{2}-32 \gamma H L_{1} \\
& -8 \beta\left\{L_{2}, L_{1}\right\}-\left(-16 \alpha \beta+\frac{176}{3} \beta\right) L_{2}+\left(\frac{176}{3} \gamma-32 \alpha \gamma\right) L_{1} \\
& +\left(32 \alpha \gamma+\frac{32}{3} \gamma\right) H+\left(\frac{32}{3} \gamma+16 \beta^{2} \alpha+\frac{32}{3} \alpha \gamma-16 \alpha^{2} \gamma-12 \beta^{2}\right)
\end{aligned}
$$

\section{Case (1c)}

$$
\begin{aligned}
{\left[R, L_{2}\right] } & =2 L_{0}^{2}-\frac{\alpha}{4} H+\alpha L_{1}+\frac{1}{8} \alpha \\
{\left[R, L_{1}\right] } & =-2\left\{L_{2}, L_{1}\right\}-L_{2} \\
R^{2} & =-\frac{2}{3}\left\{L_{2}, L_{2}, L_{1}\right\}-\frac{11}{3} L_{2}^{2}-\alpha L_{1}^{2}+\alpha H L_{1}+\frac{11}{12} \alpha L_{1}-\frac{1}{12} \alpha H \\
& +\frac{\alpha}{4}\left(\beta^{2}+\frac{1}{6}\right)
\end{aligned}
$$

\section{Case (1d)}

$$
\begin{aligned}
& {\left[R, L_{1}\right]=-2\left\{L_{1}, L_{2}\right\}+2 \alpha L_{1}-(2 \gamma+2 \beta+1) L_{2}+\left(2 \alpha \beta+\frac{1}{2} \alpha\right)} \\
& {\left[R, L_{2}\right]=6 L_{1}^{2}+2 L_{2}^{2}-4 H L_{1}+\frac{1}{2} H+(2 \beta+2 \gamma-3) L_{1}-2 \alpha L_{2}}
\end{aligned}
$$




$$
\begin{aligned}
& -(\beta+\gamma+2 \beta \gamma) \\
R^{2} & =-4 L_{1}^{3}-\frac{2}{3}\left(L_{2}, L_{2}, L_{1}\right\}+4 L_{1}^{2} H+(11-2 \beta-2 \gamma) L_{1}^{2} \\
& +\left(-\frac{11}{3}-2 \beta-2 \gamma\right) L_{2}^{2}-\frac{11}{3} H L_{1}+\alpha\left\{L_{1}, L_{2}\right\} \\
& +2\left(\frac{22}{3}(\beta+\gamma)+4 \beta \gamma\right) L_{1}+\left(\frac{11}{3} \alpha+4 \beta \alpha\right) L_{2}+\left(-\frac{1}{6}-\frac{3}{2} \gamma-4 \beta \gamma-\frac{3}{2} \beta\right) H \\
& +\left(-\frac{1}{6} \beta-\frac{1}{6} \gamma+\frac{3}{4} \beta^{2}+\frac{3}{4} \gamma^{2}-2 \beta \alpha^{2}+2 \beta^{2} \gamma+\frac{13}{6} \beta \gamma-\frac{3}{4} \alpha^{2}+2 \beta \gamma^{2}\right) .(60)
\end{aligned}
$$

\section{Case (2)}

$$
\begin{aligned}
{\left[R, L_{2}\right] } & =-8 c^{4} H^{2}-8\left(2 c^{4}-1\right) L_{2}^{2}+16 c^{2}\left(2 c^{2}-1\right) H L_{2}+8\left\{L_{2}, L_{1}\right\} \\
& +16 c^{2}\left(2 c^{2}-1\right) H+8\left(4 \gamma c^{2}+1-2 c^{4}\right) L_{2}+16 c^{4} L_{1}+8\left[(\alpha-\beta)^{2}+4 \gamma\right] c^{4} \\
{\left[R, L_{1}\right] } & =-8\left(c^{2}-1\right)\left(5 c^{2}+1\right) c^{2} H^{2}-24 c^{2}\left(c^{4}-1\right) L_{2}^{2}-8 c^{2} L_{1}^{2} \\
& +32 c^{2}\left(c^{2}-1\right)\left(2 c^{2}+1\right) H L_{2}-16 c^{2}\left(2 c^{2}-1\right) H L_{1}+8\left(2 c^{2}-1\right)\left\{L_{2}, L_{1}\right\} \\
& +16 c^{2}\left(2 c^{6}-c^{4}-4 c^{2} \gamma-c^{2}+4 \gamma+1\right) H+16\left(-c^{8}+c^{4}+4 c^{4} \gamma-4 \gamma-1\right) L_{2} \\
& +8 c^{2}\left(-4 \gamma+2 c^{4}-1\right) L_{1}+8 c^{2}\left[\left(c^{2}(\alpha-\beta)\right)^{2}-(\alpha+\beta)^{2}+4 c^{4} \gamma\right] \\
R^{2} & =32 c^{4}\left(c^{2}-1\right) H^{3}-16 c^{2}\left(c^{4}-1\right) L_{2}^{3}-\frac{16}{3} c^{2}\left\{L_{1}, L_{1}, L_{2}\right\} \\
& +32 c^{2}\left(c^{2}-1\right)\left(2 c^{2}+1\right) L_{2}^{2} H+\frac{8}{3}\left(2 c^{2}-1\right)\left\{L_{2}, L_{2}, L_{1}\right\} \\
& -16\left(c^{2}-1\right)\left(5 c^{2}+1\right) c^{2} H^{2} L_{2}+16 c^{4} H^{2} L_{1} \\
& -16 c^{2}\left(2 c^{2}-1\right)\left\{L_{2}, L_{1}\right\}-\frac{176}{3} c^{4}\left(2 c^{2}-1\right)^{2} H^{2} \\
& +\left(64 \gamma\left(c^{4}-1\right)-\frac{176}{3}\left(c^{8}+c^{4}-1\right)\right) L_{2}^{2}-\frac{176}{3} c^{4} L_{1}^{2} \\
& +\left(-128 \gamma c^{2}\left(c^{2}-1\right)+\frac{352}{3} c^{2}\left(2 c^{6}-c^{4}-c^{2}-1\right)\right) H L_{2} \\
& +\left(-32 \gamma c^{2}+\frac{88}{3} c^{2}\left(2 c^{4}-1\right)\right)\left\{L_{2}, L_{1}\right\}+\left(-16 c^{4}(\beta-\alpha)^{2}-\frac{704}{3} c^{2} \gamma\right) L_{1} \\
& +\left(16 c^{2}\left[\left(c^{2}(\alpha-\beta)\right)^{2}-(\alpha+\beta)^{2}\right]+\frac{704}{6} c^{2} \gamma\right) L_{2} \\
& +\left(-32 c^{4}(\beta-\alpha)\left(c^{2}(\beta-\alpha)+\beta+\alpha\right)+\frac{32}{3} c^{4}-\frac{128}{3}\left(11 c^{2}-5\right) c^{4} \gamma\right) H \\
& 64 c^{4} \gamma(\beta-\alpha)^{2}+\frac{32}{3} c^{4} \alpha^{2}-256 \gamma^{2} c^{4}+\frac{64}{3} c^{4} \gamma+\frac{32}{3} c^{4} \beta^{2} . \\
& \\
& \\
&
\end{aligned}
$$


Case (3)

$$
\begin{aligned}
{\left[L_{2}, R\right] } & =-6 L_{1}^{2}-8 \gamma H+\beta H+8 \alpha L_{1}-2\left(\alpha^{2}+3 \gamma\right) \\
{\left[L_{1}, R\right] } & =-16 \gamma L_{2}-2 \beta L_{1}+2 \alpha \beta \\
R^{2} & =-4 L_{1}^{3}+16 \gamma L_{2}^{2}+8 \alpha L_{1}^{2}-16 \gamma H L_{1}+2 \beta\left\{L_{2}, L_{1}\right\}+4 \alpha \beta L_{2} \\
& -\left(44 \gamma+4 \alpha^{2}\right) L_{1}-\beta^{2} H+\left(-\frac{3}{4} \beta^{2}+32 \gamma \alpha\right) .
\end{aligned}
$$

We note that the quadratic relations in the quantum case provide useful information relating the special functions that occur as (separable) eigenfunctions for each superintegrable case $[19,20]$. For other applications of superintegrability on the real sphere or the real hyperboloid see $[17,18,19,20]$.

\section{Conclusions}

In this paper we have used the concept of a "nondegenerate potential" to add structure to the study of superintegrable classical and quantum mechanical systems on the complex 2-sphere. We have shown how to classify all such systems in a straightforward manner, so that gaps can be avoided. Furthermore, we have shown the following:

1. Each system is associated with a pair of constants of the motion in the classical case, and a pair of symmetry operators in the quantum case, that generate a quadratic algebra.

2. There is a one-to-one correspondence between superintegrable systems and free-field symmetry operators that generate quadratic algebras.

3. Superintegrability implies multiseparability, i.e., separability in more than one coordinate system.

\section{Appendix}

As is well known $[14,21]$ there are essentially five coordinate systems on the complex 2-sphere in which the free particle Hamilton-Jacobi equation 
separates: spherical, elliptic, horospherical, and degenerate elliptic of the first and second kinds. We describe these coordinate systems and their corresponding free particle constants of the motion $L$. (We adopt the basis $J_{1}=-z p_{y}, \quad J_{2}=z p_{x}, \quad J_{3}=x p_{y}-y p_{x}$, for the Lie algebra $s o(3, C)$, where $z=\sqrt{1-x^{2}-y^{2}}$.) The systems are:

\section{Spherical Coordinates}

$$
\begin{aligned}
x=\sin \theta \cos \varphi, & y=\sin \theta \sin \varphi \\
z=\cos \theta, & L=J_{3}^{2}
\end{aligned}
$$

\section{Elliptic Coordinates}

$$
\begin{aligned}
x^{2}=\frac{(r u-1)(r v-1)}{1-r}, & y^{2}=\frac{r(u-1)(v-1)}{1-r} \\
z^{2}=r u v, & L=J_{1}^{2}+r J_{2}^{2}
\end{aligned}
$$

\section{Horospherical Coordinates.}

$$
\begin{aligned}
x=\frac{i}{2}\left(v+\frac{u^{2}-1}{v}\right), & y=\frac{1}{2}\left(v+\frac{u^{2}+1}{v}\right), \\
z=\frac{i u}{v}, & L=\left(J_{1}+i J_{2}\right)^{2}
\end{aligned}
$$

\section{Degenerate Elliptic Coordinates of Type 1}

$$
\begin{aligned}
x+i y=\frac{4 c u v}{\left(u^{2}+1\right)\left(v^{2}+1\right)}, \quad x-i y & =\frac{\left(u^{2} v^{2}+1\right)\left(u^{2}+v^{2}\right)}{c u v\left(u^{2}+1\right)\left(v^{2}+1\right)}, \\
z=\frac{\left(u^{2}-1\right)\left(v^{2}-1\right)}{\left(u^{2}+1\right)\left(v^{2}+1\right)}, \quad L & =\left(J_{1}+i J_{2}\right)^{2}-c^{2} J_{3}^{2}
\end{aligned}
$$

\section{Degenerate Elliptic Coordinates of Type 2}

$$
\begin{aligned}
x+i y & =-i u v, \quad x-i y=-\frac{\left(u^{2}+v^{2}\right)^{2}}{4}, \\
z & =\frac{i}{2} \frac{u^{2} v^{3}}{u v}, \quad L=J_{3}\left(J_{1}-i J_{2}\right)
\end{aligned}
$$




\section{References}

[1] N.W.Evans. Superintegrability in Classical Mechanics; Phys.Rev. A 41 (1990) 5666; Group Theory of the Smorodinsky-Winternitz System; J.Math.Phys. 32, 3369 (1991).

[2] N.W.Evans. Superintegrability of the Calogero-Moser System. Phys. Lett. A 95, 279 (1983); Super-Integrability of the Winternitz System; Phys.Lett. A 147, 483 (1990).

[3] L.P.Eisenhart. Enumeration of Potentials for Which One-Particle Schrödinger Equations Are Separable; Phys.Rev. 74, 87 (1948).

[4] J.Friš, V.Mandrosov, Ya.A.Smorodinsky, M.Uhlir and P.Winternitz. On Higher Symmetries in Quantum Mechanics; Phys.Lett. 16, 354 (1965).

[5] J.Friš, Ya.A.Smorodinskii, M.Uhlír and P.Winternitz. Symmetry Groups in Classical and Quantum Mechanics; Sov.J.Nucl.Phys. 4, 444 (1967).

[6] A.A.Makarov, Ya.A.Smorodinsky, Kh.Valiev and P.Winternitz. A Systematic Search for Nonrelativistic Systems with Dynamical Symmetries; Nuovo Cimento A 52, 1061 (1967).

[7] D.Bonatos, C.Daskaloyannis and K.Kokkotas. Deformed Oscillator Algebras for Two-Dimensional Quantum Superintegrable Systems; Phys. Rev. A 50, 3700 (1994).

[8] F.Calogero. Solution of a Three-Body Problem in One Dimension; J.Math.Phys. 10, 2191 (1969).

[9] A.Cisneros and H.V.McIntosh. Symmetry of the Two-Dimensional Hydrogen Atom; J.Math.Phys. 10, 277 (1969).

[10] L.G.Mardoyan, G.S.Pogosyan, A.N.Sissakian and V.M.Ter-Antonyan. Elliptic Basis for a Circular Oscillator. Nuovo Cimento, B 88, 43 (1985), Two-Dimensional Hydrogen Atom: I. Elliptic Bases; Theor.Math.Phys. 61, 1021 (1984); Hidden symmetry, Separation of Variables and Interbasis Expansions in the Two-Dimensional Hydrogen Atom. J.Phys., A 18, 455 (1985). 
[11] B.Zaslow and M.E.Zandler. Two-Dimensional Analog of the Hydrogen Atom; Amer. J.Phys. 35, 1118 (1967).

[12] Ya.A.Granovsky, A.S.Zhedanov and I.M.Lutzenko. Quadratic Algebra as a 'Hidden' Symmetry of the Hartmann Potential. J.Phys. A 24, 3887 (1991).

[13] P.Letourneau and L.Vinet. Superintegrable systems: Polynomial Algebras and Quasi-Exactly Solvable Hamiltonians. Ann. Phys. 243, 144$168,(1995)$.

[14] W.Miller, Jr. Symmetry and Separation of Variables. Addison-Wesley Publishing Company, Providence, Rhode Island, 1977.

[15] E.G.Kalnins, W.Miller Jr. and G.S.Pogosyan. Completeness of multiseparable superintegrability in $E_{2, C}$. (submitted 2000).

[16] M.F. Rañanda and M.Santander. Superintegrable systems on the twodimensional sphere $S 2$ and the hyperbolic plane $H 2$ J.Math.Phys. 40, 5026 (1999).

[17] C.P.Boyer, E.G.Kalnins and P.Winternitz. Completely integrable relativistic Hamiltonian systems and separation of variables in Hermitian hyperbolic spaces. J.Math.Phys. 24, 2022 (1983).

[18] C.Grosche, G.S.Pogosyan, A.N.Sissakian. Path Integral Approach to Superintegrable Potentials. The Two-Dimensional Hyperboloid. Phys.Part.Nucl. 27, 244, (1996).

[19] E.G.Kalnins, W.Miller Jr. and G.S.Pogosyan. Superintegrability and associated polynomial solutions. Euclidean space and the sphere in two dimensions; J.Math.Phys. 37, 6439, (1996).

[20] E.G.Kalnins, W.Miller Jr. and G.S.Pogosyan. Superintegrability on the two dimensional hyperboloid; J.Math.Phys. 38, 5416, (1997).

[21] E.G. Kalnins, Separation of Variables for Riemannian Spaces of Constant Curvature, Pitman, Monographs and Surveys in Pure and Applied Mathematics 28, Longman, Essex, England, 1986. 